\title{
Material Point Methods Applied to One-dimensional Shock Waves and Dual Domain Material Point Method with Sub-points
}

\author{
Tilak R. Dhakal and Duan Z. Zhang \\ Theoretical Division, Fluid Dynamics and Solid Mechanics Group (T-3, B216) \\ Los Alamos National Laboratory \\ Los Alamos, NM 87545, USA
}

\begin{abstract}
Using a simple one-dimensional shock problem as an example, the present paper investigates numerical properties of the original material point method (MPM), the generalized interpolation material point (GIMP) method, the convected particle domain interpolation (CPDI) method, and the dual domain material point (DDMP) method.

For a weak isothermal shock of ideal gas, the MPM cannot be used with accuracy. With a small number of particles per cell, GIMP and CPDI produce reasonable results. However, as the number of particles increases the methods fail to converge and produce pressure spikes. The DDMP method behaves in an opposite way. With a small number of particles per cell, DDMP results are unsatisfactory. As the number of particles increases, the DDMP results converge to correct solutions, but the large number of particles needed for convergence makes the method very expensive to use in these types of shock wave problems in two- or three-dimensional cases.

The cause for producing the unsatisfactory DDMP results is identified. A simple improvement to the method is introduced by using sub-points. With this improvement, the DDMP method produces high quality numerical solutions with a very small number of particles.
\end{abstract}

Although in the present paper, the numerical examples are one-dimensional, all derivations are for multidimensional problems. With the technique of approximately tracking particle domains of CPDI, the extension of this sub-point method to multidimensional problems is straightforward. This new method preserves the conservation properties of the 
DDMP method, which conserves mass and momentum exactly and conserves energy to the second order in both spatial and temporal discretizations.

Keywords: Material point methods, Shock waves.

\section{Introduction}

Since its first introduction [1,2], the material point method has been used in many problems involving large material deformations $[3,4,5,6,7,8]$ in which a traditional finite element method encounters difficulties due to mesh or element distortion. Although both the finite element method and the material point method seek approximate weak solutions to the partial differential equations, there are two significant differences that result in different numerical properties of the methods. The first difference is that the finite element method is a pure Lagrangian method in which elements move and deform with the material, while the material point method uses an arbitrary Eulerian mesh, which may stay fixed during a material motion and avoids the mesh or element distortion in cases of large material deformation. The Lagrangian capability of the material point method resides in its use of material points to carry history dependent quantities.

The second difference is the numerical integration method used to approximate the inner product of two functions. In the finite element method the integration for the internal force calculation is often calculated using the Gauss method with Gauss integration points specified at given logical coordinates of the element for the purpose of accuracy. In a material point method, the material points are used not only to carry important field quantities, such as stress and damage of the material, but also used as integration points. Unlike in the finite element method, we do not have control on locations of these material points. In a material point method, the numerical integration in the internal force calculation is approximated by a low accuracy Riemann sum.

Comparing to the finite element method, the freedom or advantage of the material point method is gained at the cost of numerical accuracy and smoothness in the internal force calculation. One problem caused by this low accuracy integration is now the well known cell crossing noise [9] of the method. The generalized interpolation material point 
method (GIMP) reduces this noise. In this version of the material point method, the concept of particle domain is introduced to perform integrations in the calculation of the nodal mass and the internal force. The smoothing effect is provided by averaging or integrating over the particle domain. To conserve momentum, the GIMP method requires that the domains of the particles cover the entire computational domain without overlap. This requirement is also called the partition of unity, since this is equivalent to requiring that the sum of the particle characteristic functions equals to unity everywhere in the computational domain. It is rather straightforward to satisfy this requirement in onedimensional problems. For two- or three-dimensional problems with a significant material deformation, it is nearly impossible to numerically satisfy this requirement. This is similar to the difficulty encountered by the finite element methods due to element distortion. To alleviate this difficulty, there are two approximate versions of the GIMP method, uGIMP and cpGIMP [9]. In uGIMP, the partition of unity requirement is simply ignored, and the particle domains are assumed unchanged during the material deformation; therefore it can only be applied to problems with small material deformations. In cpGIMP, the particle domains are assumed to be rectangles all the time. Only deformations caused by the diagonal components of the deformation gradient are considered in the change of the particle domain. Therefore, the method cannot be used for problems with strong shear or rotation.

To improve this situation, another version of the material point method, called the convected particle domain interpolation (CPDI) [10] method, allows significant rotation and shear deformation by using parallelograms (in two-dimension) and parallelepipeds (in three-dimension) to cover the computational domain. The parallelograms or parallelepipeds can move, rotate, and deform according to the velocity and the velocity gradient at the center. In this way, the CPDI method provides a local linear approximation to the deformation field in the calculation of the particle domains, but gaps and overlaps still happen among the deformed parallelograms and parallelepipeds.

Both the GIMP and CPDI methods rely on averages over particle domains to provide 
the smoothing effect in reducing the numerical noise caused by cell crossing of particles. In these methods, the use of particle domain introduces a new numerical length scale in addition to the mesh size and has numerical consequences. For instance, if the domain size is reduced, the smoothing effects are also reduced. As we show in the following section these methods encounter convergence issues as the number of particles is increased.

To eliminate the cell crossing noise, the third version of the improved material point method, called the dual domain material point (DDMP) method, uses an equivalent stress field to calculate the internal force. The equivalent stress is constructed using the stress at the material points and is only different from the original stress field by an amount of order of $(\Delta x)^{2}$ in the sense of weak solution, where $\Delta x$ is the typical mesh size. The internal force calculated using such a stress field is a linear combination of the products of the particle volumes and the stresses, and the coefficients in this linear combination can be then regarded as the modified gradient of the shape function. The equivalent stress field is constructed in such a way that the corresponding modified gradient of the shape function is continuous on cell boundaries. Unaltered from the original MPM, material points in the DDMP method serve two roles: as Lagrangian markers carrying history information and as integration points. These two roles of material points are separated in the DDMP method improved with sub-points introduced in Section 4. In this new method the material points or particles are used to carry physical quantities and history information, while the numerical integration is done by using the sub-points generated at each time step. This improved DDMP method preserves the conservation properties of the DDMP method without encountering issues of partition of unity as in the GIMP and CPDI methods. Mass and momentum are conserved exactly, and energy conservation is second order both in spatial and time discretizations.

To investigate properties of different versions of the material point method, we use them to calculate the propagation of one-dimensional weak isothermal shock waves in an ideal gas. Although this problem is very simple and even unphysical, it actually reveals many numerical properties of the MPM methods. The main objective of the present paper 
is to improve the material point method by exploiting advantages of different versions of the material point method, while avoiding their disadvantages. At the end of the paper, we demonstrate that the improved method can also be used in strong adiabatic shocks to produce high quality results.

\section{Material Point Methods}

Material point methods seek weak solutions of partial differential equations. For a dynamic problem the discretized momentum equation can be written as $[2,11]$,

$$
\sum_{j=1}^{N} m_{i j} \frac{d \boldsymbol{v}_{j}}{d t}=\boldsymbol{f}_{i}^{i n t}+\int_{\Omega} \rho \boldsymbol{b} S_{i} d v+\int_{\partial \Omega} \boldsymbol{\sigma} \cdot \boldsymbol{n} S_{i}(\boldsymbol{x}) d A,
$$

with the internal force defined as

$$
\boldsymbol{f}_{i}^{i n t}=-\int_{\Omega} \boldsymbol{\sigma} \cdot \nabla S_{i} d v,
$$

where $N$ is the number of nodes in the computational domain, $m_{i j}$ the mass matrix element for nodes $i$ and $j, \boldsymbol{v}_{j}$ the velocity at the node $j, \Omega$ the computational domain, $\rho$ the density, $\boldsymbol{b}$ the body force, $\boldsymbol{\sigma}$ the stress, $S_{i}$ the shape function of node $i, \boldsymbol{n}$ the outward normal on the boundary of the computational domain, $d A$ an element of surface area, and $d v$ the volume element in the computational domain $\Omega$. In most calculations the mass matrix can be replaced by a diagonal matrix with the diagonal element being the sum of the elements in the same row [12].

In the finite element method, the force is calculated using the Gauss integration points. In the MPM method the internal force is calculated simply as a Riemann sum of the material points [2],

$$
\boldsymbol{f}_{i}^{i n t} \approx-\sum_{p=1}^{N_{p}} \boldsymbol{\sigma}\left(\boldsymbol{x}_{p}, t\right) v_{p} \cdot \nabla S_{i}\left(\boldsymbol{x}_{p}\right)
$$

where $\boldsymbol{x}_{p}$ is the material point location, $N_{p}$ is the number of particles in the computational domain, and $v_{p}$ is the volume of the material point.

While this approximation has been successfully used in many MPM calculations, mostly for solid materials, we now show this approximation fails if we use it to calculate a small 
perturbation of an ideal gas under isothermal condition, in which material points do not move across cells. Suppose a one-dimensional pipe initially separated by a partition located at node $j$ in Fig. 1. To the left of the partition the gas pressure is slightly higher than that to the right. The gas on the both sides of the partition is at rest initially. At time $t=0$ the partition is suddenly removed. Physically we expect to see a weak shock and expansion waves to propagate either direction in the computational domain. However if we use approximation (3), we have

$$
\boldsymbol{f}_{i}^{i n t} \approx \sum_{p=1}^{N_{p}} v_{p} P_{p} \nabla S_{i}
$$

where $P_{p}$ is the pressure $P$ at material point $p$. In an isothermal case, $v_{p} P_{p}$ equals to two constants $C_{L}$ or $C_{R}$ respectively for the particles initially located at the left or right of the partition. Since $\nabla S_{i}$ is piecewise constant, for particle distributions shown in Fig. 1 only node $j$, the location of initial partition, experiences a net non-zero force. The net force at all other nodes is exactly zero. As a result, only node $j$ acquires an acceleration to the right, while all other nodal accelerations remain zero. Then only particles in cells between nodes $j-1$ to $j+1$ obtain velocity increases, while other particle velocities remain zero, according to node-particle velocity relation

$$
\boldsymbol{v}_{p}^{n+1}=\boldsymbol{v}_{p}^{n}+\sum_{i=1}^{N} S_{i}\left(\boldsymbol{x}_{p}^{n}\right) \frac{d \boldsymbol{v}_{i}}{d t} \Delta t,
$$

where the superscripts denote the time step. After the particles move according to the velocity interpolated from nodes, their velocities are mapped to nodes using

$$
\boldsymbol{v}_{i}^{n+1}=\frac{\sum_{p=1}^{N_{p}} m_{p} v_{p}^{n+1} S_{i}\left(\boldsymbol{x}_{p}^{n+1}\right)}{\sum_{p=1}^{N_{p}} m_{p} S_{i}\left(\boldsymbol{x}_{p}^{n+1}\right)} .
$$

Only nodes $j-1, j$, and $j+1$ achieve nonzero velocities, while other nodal velocities are zero. When these nodal velocities are used to calculate the stress or pressure at the

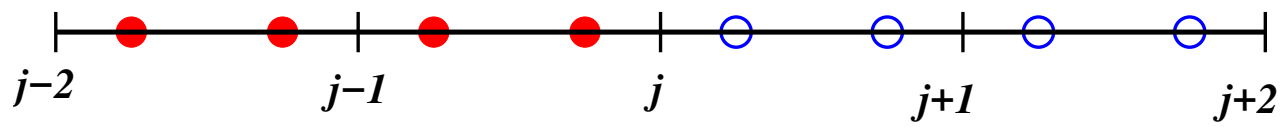

Figure 1: Particles and cells in a one-dimensional weak shock calculation. 
particles, only the pressure between these nodes $j-2$ and $j+2$, the four cells on both sides of $j$ as shown in Fig. 2 with $j=0$, are changed, while other particle pressures remain unaltered. As time advances, the velocities at node $j$ and the two neighboring nodes continue to increase, while other nodal velocities remain zero. The waves fail to propagate. During this time the particle pressures in the two cells left of node $j$ continue to decrease and even become less than the initial value on the right. Similarly, the particle pressures in the two cells right of node $j$ continue to increase and exceed the initial value on the left. The resulting high pressures at the particles on the right cannot push nodal velocity back because in this isothermal case, the particle volume is reduced, and $v_{p} P_{p}=C_{R}$. Similarly the resulting low pressures at the left particles cannot pull nodal velocity back because $v_{p} P_{p}=C_{L}$. Since $C_{L}>C_{R}$, the nodal force from (4) is always positive for node $j=0$, and the process accelerates. This process is shown in Fig. 2 for particle pressure values at different times.

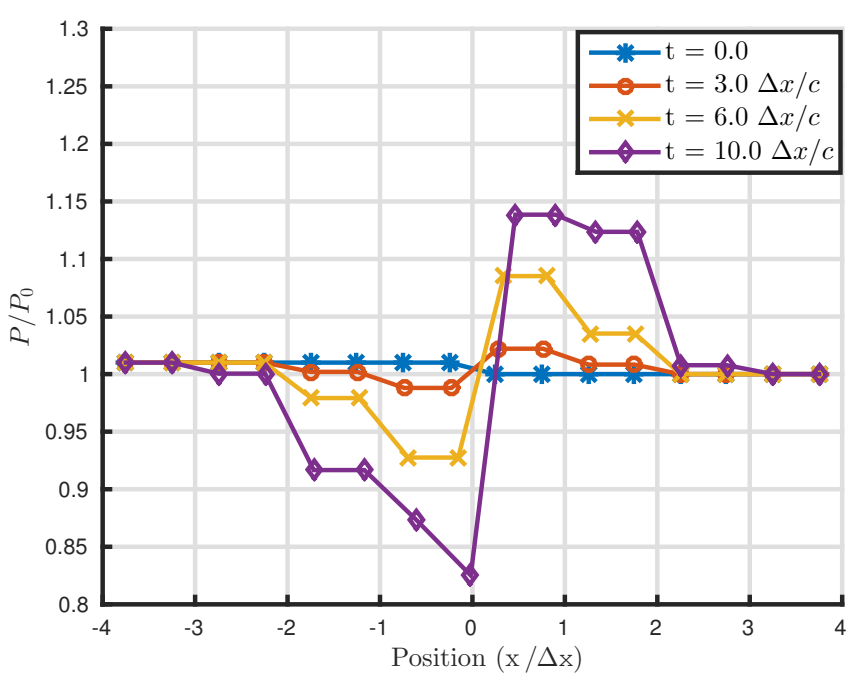

Figure 2: Particles pressure near one-dimensional shock front. Position 0 corresponds node $j$ in Fig. 1.

This process continues until the first particle left of the partition moves into the right cell, as shown in Fig. 2 at $t=10 \Delta x / c$, where $c$ is the speed of sound. At that time the forces on nodes $j-1$ and $j+1$ become nonzero, and then the particle pressures in the next neighboring cells start being affected. However this propagation of the disturbance is 
purely numerical, is not continuous, occurs only after the particle has been displaced by a quarter of the cell length for the particle arrangement shown in Fig. 1, and is too late for a proper propagation of the waves.
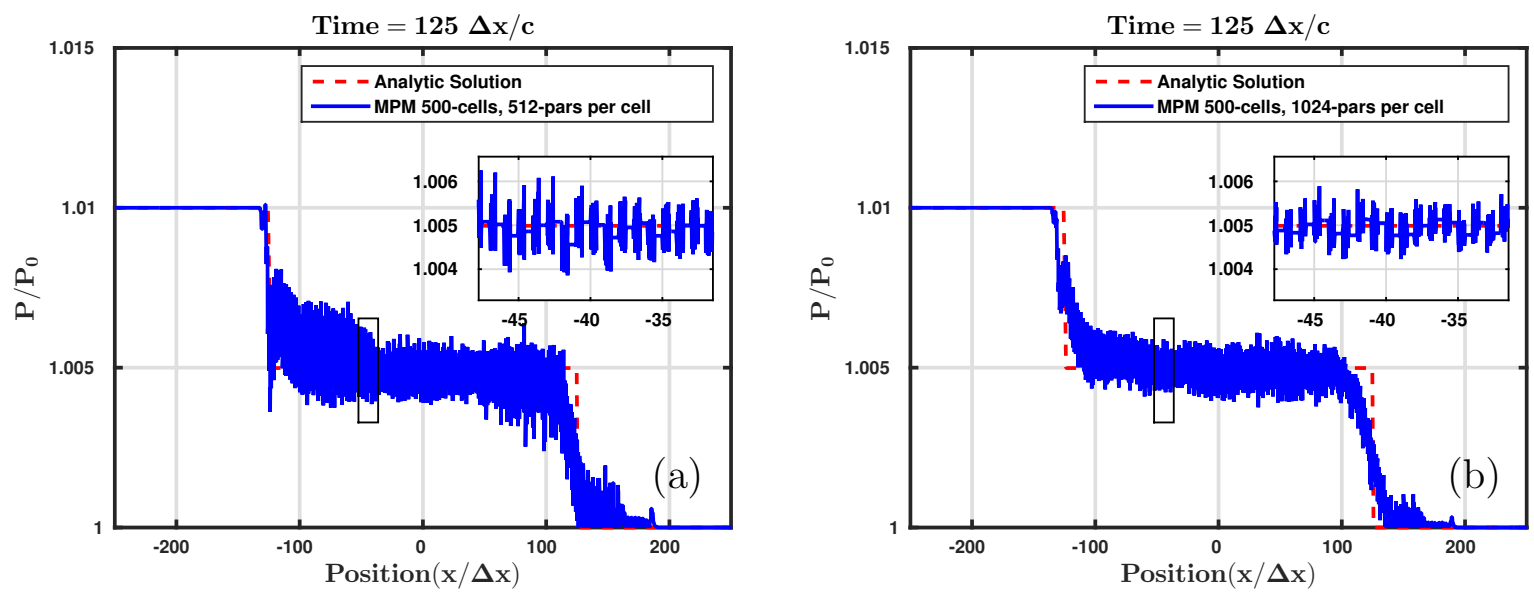

Figure 3: Particle pressure calculated with MPM method using (a) 512 particles per cell, (b) 1024 particles per cell.

Despite the incorrect wave propagation results explained above, the process of material point motion resembles material motion in the physical situation: as time advances, some of the material in the left cell moves across the nodes into the cell on the right to cause a pressure change. In the MPM method, because the mass is lumped at material points, this cell crossing motion does not occur until the material point moves across a cell. One might think that if sufficient number of material points are used, the cell crossing can happen earlier so that the pressure disturbance can propagate to the other nodes earlier and the pressure inversion can be avoided. This physical intuition is correct. However, the use of many material points results in noise and pressure spikes as shown in Fig. 3 calculated using 512 and 1024 particles per cell in the initial particle placement with time step $0.1 \Delta x / c$. In the figure the results roughly approximate the correct behavior. The numerical quality is apparently unsatisfactory, although the results are slightly better with the larger amount of particles.

The spike is caused by cell crossing of the particles. Suppose one particle moves from 
a high pressure cell to a low pressure cell carrying a high pressure value with it. While this new member in the low pressure cell contributes to the resistance to the compression of the low pressure cell, the majority of particles in the cell are still at low pressure. The cell is still being compressed causing a pressure increase for particles in the low pressure cell, including the new member carrying a high pressure value. This trend is correct for most of the particles in the cell, but for the particle just arrived from the high pressure cell, this compression further increases its pressure, instead of relieving it. Meanwhile, the upstream particles remaining in the high pressure cell experience a pressure release from a positive velocity divergence in the cell. The particle that just moved into the low pressure cell experiences a further pressure increase, while its upstream neighbors experience a pressure release, and its downstream neighbors are still at their low pressure values. Thus, the particle that just entered the low pressure cell has a higher pressure value than its neighbors, and it generates a pressure spike.

These pressure spikes are caused by particles moving across cell boundaries, and currently there are three versions of the material method, GIMP, CPDI and DDMP available to reduce the cell crossing noise. We now use them to see how they perform in this case of one-dimensional shock propagation. 

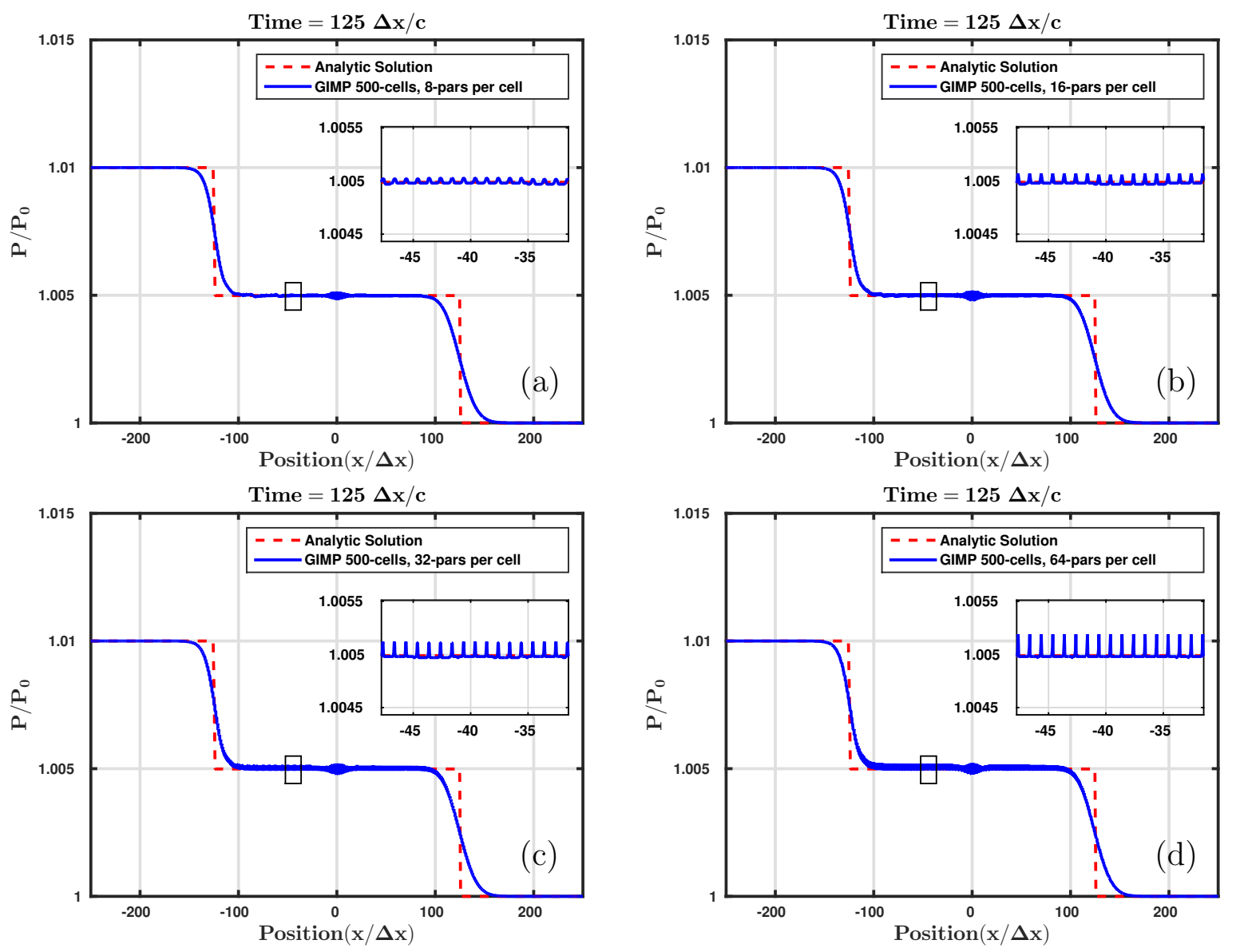

Figure 4: Particle pressure calculated with GIMP method using (a) 8 particles per cell, (b)16 particles per cell, (c) 32 particles per cell, and (d) 64 particles per cell.

The results of GIMP are shown in Fig. 4. There is a significant improvement compared to the original MPM results. Instead of needing a few hundred particles per cell to show just the rough shape of the results as in Fig.3, the GIMP results obtained using 8 particles per cell in the initial particle placement result in acceptable results compared to the analytic solution. However, the pressure spikes are evident as more particles per cell are used. Fig. 4 shows results calculated using 8, 16, 32, and 64 particles per cell in the initial particle placement. This behavior of the solution is qualitatively similar for the CPDI method as shown in Fig. 5. The improvement of these two methods over the original MPM comes from the introduction of finite particle size. In the internal force calculation, instead of using the gradient of the shape function evaluated directly at a particle location, 
which abruptly changes by $2 / \Delta x$ in a time step in this one-dimensional case as the particle moves across a cell boundary in the original MPM, the GIMP and CPDI methods replace this discontinuous value of the shape function gradient at a material point by its average over the finite particle domain. With this replacement, for a particle near a cell boundary, the change in the value of the modified shape function gradient in a time step is limited by $2\left|\boldsymbol{v}_{p}\right| \Delta t /\left(\Delta x \ell_{p}\right)$, if $\left|\boldsymbol{v}_{p}\right| \Delta t \leq \ell_{p}$, where $\ell_{p}$ is the particle length in this one-dimensional case. These methods require that, at least initially, the particle domains cover the entire computational domain without gap or overlap. As the number of material points per cell increases, the size $\ell_{p}$ of the particle is reduced, and change in the modified shape function gradient in a time step is increased. Although the use of a large number of particles increases the numerical integration accuracy in the internal force calculation, the effect of this accuracy increase is very limited because the use of small number of particles already produces reasonable results as shown in Figs. 4 and 5. The pressure spikes are not caused by the lack of the integration accuracy, but rather by the discontinuity or rapid change of the value of the shape function gradient in a time step. When the particle size $\ell_{p}$ becomes less than $\left|\boldsymbol{v}_{p}\right| \Delta t$, or the finite-domain particle moves across a cell boundary completely in a time step, the change becomes to $2 / \Delta x$, the same value as in the original MPM, and the benefit of using a finite particle domain is lost completely. In other words, the introduction of finite particle domain also introduces a concept of the particle Courant number, $\left|\boldsymbol{v}_{p}\right| \Delta t / \ell_{p}$, based on the particle size. The benefit of GIMP and CPDI methods reduces with increasing particle Courant number. Therefore, as the number of particles per cell increases, the pressure spikes increase. The appearance of the spikes at large $(>8)$ particles per cell might not be a significant issue for this problem with a small deformation, because one can always use a small number of particles per cell, but this is a special case of isothermal shock. For problems with large deformation, however, we do not have control on the number of particles per cell, and particles can aggregate in certain regions of the computational domain. 

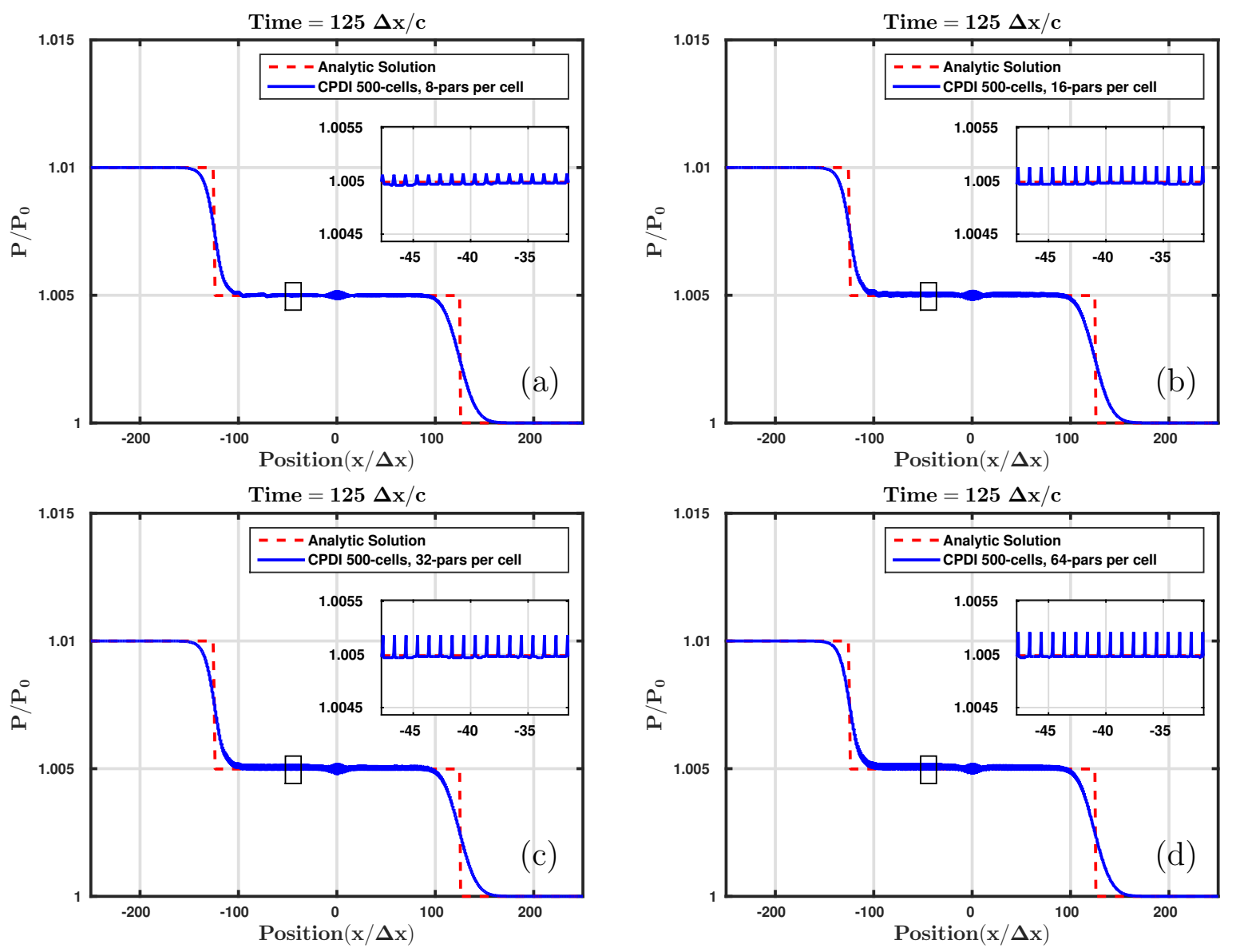

Figure 5: Particle pressure calculated with CPDI method using (a) 8 particles per cell, (b) 16 particles per cell, (c) 32 particles per cell, and (d) 64 particles per cell.

This weak shock problem is also calculated using the DDMP method, as shown in Fig. 6. With 8 particles per cell in the initial particle placement, the results show spurious oscillation in Fig. 6(a) and are worse than the GIMP and CPDI methods. But different from the GIMP and CPDI methods, DDMP results improve as the number of particles increases. 

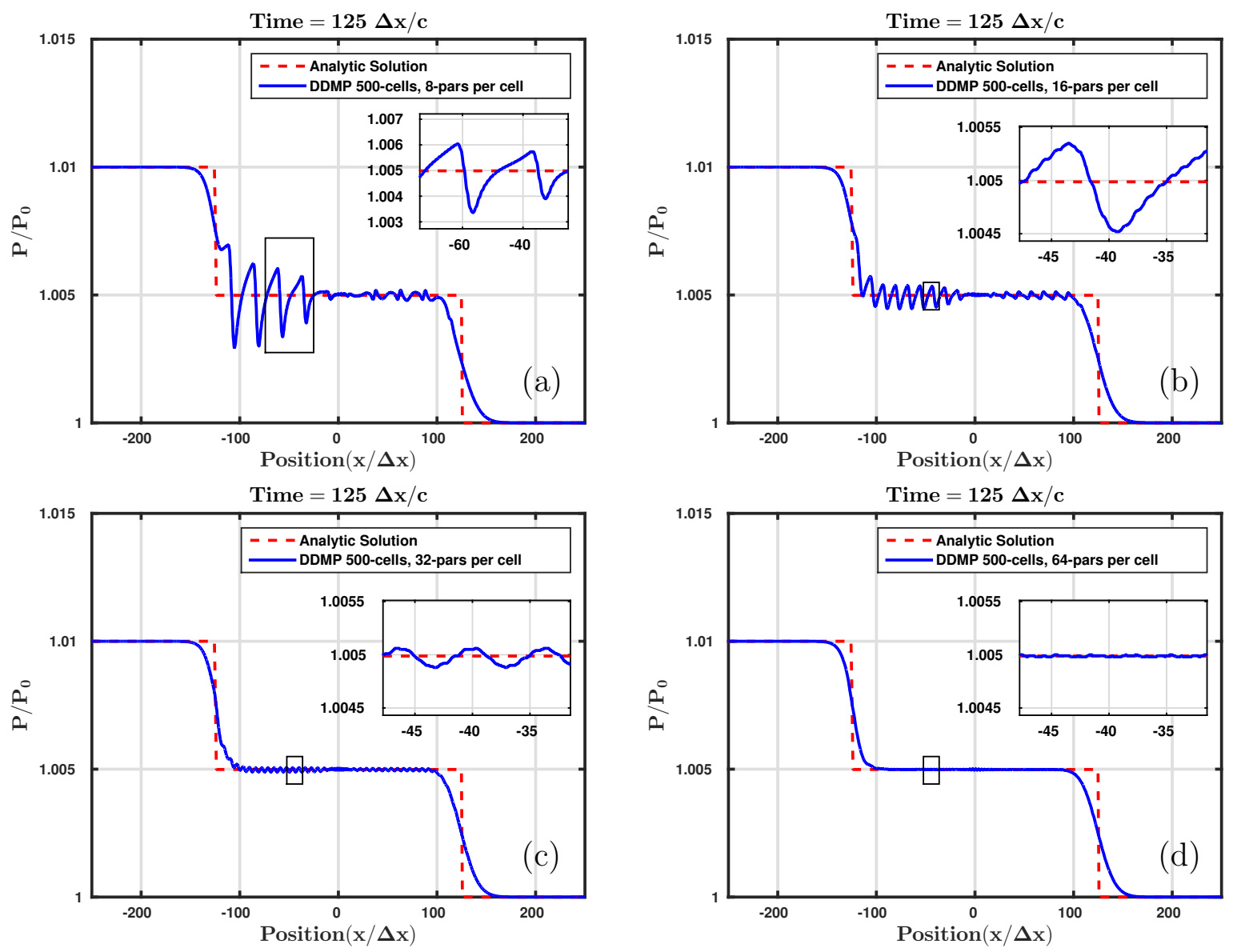

Figure 6: Particle pressure calculated with DDMP method using (a) 8 particles per cell, (b) 16 particles per cell, (c) 32 particles per cell, and (d) 64 particles per cell.

From these results calculated using different versions of the material point method, we conclude that

1. The original MPM cannot be used for this weak shock propagation problem.

2. The finite-sized particle domains used in GIMP and CPDI methods provide smoothing effects, and therefore, when a small number of particles are used, the GIMP and CPDI methods give reasonable results for this weak shock problem. But these methods can only benefit calculations with a sufficiently small particle Courant number, and fail as number of particles increases.

3. The DDMP method is inaccurate when a small number of particles are used, but improves as the number of particles increases. However, the large number of particles 
required to achieve accurate results renders this method very expensive, especially for similar problems in two- or three- dimensional cases.

Since the DDMP method is the only one among the four versions of the material point method that converges to the correct solution with increasing particle number, in the rest of this paper we focus on improvements to the DDMP method.

\section{Error Analysis for the DDMP Method}

Instead of introducing the concept of finite particle domain as in the GIMP and CPDI methods, the DDMP method reduces the cell crossing noise by adding an auxiliary stress

$$
\boldsymbol{\sigma}_{A}=A(\boldsymbol{x}, t)+\sum_{j} \frac{S_{j}(\boldsymbol{x})}{V_{j}} \sum_{p=1}^{N_{p}} v_{p}(t) \boldsymbol{\sigma}_{p}(t) S_{j}\left(\boldsymbol{x}_{p}\right)-\boldsymbol{\sigma}(\boldsymbol{x}, t)
$$

to the stress $\boldsymbol{\sigma}$ in (2) to improve the numerical properties of the integral, where

$$
\boldsymbol{A}(\boldsymbol{x})=\sum_{p=1}^{N_{p}} \alpha\left(\boldsymbol{x}_{p}\right) v_{p} \boldsymbol{\sigma}_{p} \delta\left(\boldsymbol{x}-\boldsymbol{x}_{p}\right)-\sum_{j=1}^{N} \frac{S_{j}(\boldsymbol{x})}{V_{j}} \sum_{p=1}^{N_{p}} \alpha\left(\boldsymbol{x}_{p}\right) v_{p} \boldsymbol{\sigma}_{p} S_{j}\left(\boldsymbol{x}_{p}\right),
$$

and $\alpha(\boldsymbol{x})$ is a continuous function whose value is zero on cell boundaries. A typical choice for $\alpha(\boldsymbol{x})$ is [13]

$$
\alpha(\boldsymbol{x})=0.5\left\{\prod_{k=1}^{n_{c}}\left[n_{c} S_{k}(\boldsymbol{x})\right]\right\}^{\frac{3}{2\left(n_{c}-1\right) d}},
$$

where $n_{c}$ is the number of nodes in the cell, and $d(=1,2,3)$ is the dimension of the problem.

To ensure that the addition of the auxiliary stress $\boldsymbol{\sigma}_{A}$ in (2) does not affect the accuracy of the numerical solution to the original equations, which is second order in $\Delta x$, one needs to ensure the auxiliary stress is of the same order. It has been proved in [13] that $\boldsymbol{A}(\boldsymbol{x})=O(\Delta x)^{2}$, independent of choice of the material points, their volumes, and function $\alpha(\boldsymbol{x})$. The proof is based on the relation

$$
h(\boldsymbol{x})=\sum_{j=1}^{N} h_{j} S_{j}(\boldsymbol{x})+O(\Delta x)^{2}=\sum_{j=1}^{N}\left[\frac{1}{V_{j}} \int_{\Omega} S_{j}(\boldsymbol{y}) h(\boldsymbol{y}) d v_{y}\right] S_{j}(\boldsymbol{x})+O(\Delta x)^{b},
$$

where $h(\boldsymbol{x})$ is a smooth function, $h_{j}$ is the value of $h(\boldsymbol{x})$ at node $j$, and $b=2$ if $\boldsymbol{x}$ is located in an interior cell, and $b=1$ if $\boldsymbol{x}$ is in a boundary cell. The first identity 
comes from the property of the shape functions, and the second relation holds because $h_{j}=\int_{\Omega} S_{j}(\boldsymbol{y}) h(\boldsymbol{y}) d v_{y} / V_{j}+O(\Delta x)^{b}$ and $V_{j}=\int_{\Omega} S_{j}(\boldsymbol{y}) d v_{y}$, or in other words, the value of $h$ at a mesh node can be approximated by the average value in the square brackets in (10).

The summation over material points in (7) can be regarded as a Riemann sum. As the maximum volume of the particles $v_{p}$ approaches zero, the sum becomes $\int_{\Omega} S_{j}(\boldsymbol{y}) \boldsymbol{\sigma}(\boldsymbol{y}, t) d v_{y}$. Using relation (10), one finds

$$
\begin{aligned}
& \lim _{\max \left(v_{p}\right) \rightarrow 0} \sum_{j} \frac{S_{j}(\boldsymbol{x})}{V_{j}} \sum_{p=1}^{N_{p}} v_{p}(t) \boldsymbol{\sigma}_{p}(t) S_{j}\left(\boldsymbol{x}_{p}\right)=\sum_{j=1}^{N}\left[\frac{1}{V_{j}} \int_{\Omega} S_{j}(\boldsymbol{y}) \boldsymbol{\sigma}(\boldsymbol{y}) d v_{y}\right] S_{j}(\boldsymbol{x}) \\
& =\boldsymbol{\sigma}(\boldsymbol{x}, t)+O(\Delta x)^{b} .
\end{aligned}
$$

Thus, we have proved that, in the sense of weak solutions, the auxiliary stress defined in (7) is second order in $\Delta x, \boldsymbol{\sigma}_{A}(\boldsymbol{x})=O(\Delta x)^{2}$, when a sufficient number of material points is used, because the total volume of the boundary cells is proportional to $\Delta x$.

After adding the auxiliary stress $\boldsymbol{\sigma}_{A}$ to the stress $\boldsymbol{\sigma}$ in (2), the force integral can be calculated as

$$
\boldsymbol{f}_{i}^{\text {int }} \approx-\int_{\Omega}\left(\boldsymbol{\sigma}+\boldsymbol{\sigma}_{A}\right) \cdot \nabla S_{i} d v=-\sum_{p=1}^{N_{p}} v_{p} \boldsymbol{\sigma}_{p} \cdot \overline{\nabla S_{i}}\left(\boldsymbol{x}_{p}\right)
$$

where

$$
\overline{\nabla S_{i}}(\boldsymbol{x})=\alpha(\boldsymbol{x}) \nabla S_{i}(\boldsymbol{x})+[1-\alpha(\boldsymbol{x})] \sum_{j=1}^{N} \frac{S_{j}(\boldsymbol{x})}{V_{j}} \int S_{j}(\boldsymbol{y}) \nabla S_{i}(\boldsymbol{y}) d v_{y},
$$

with $N$ being the number of the nodes in the computational domain. In this way, one can also regard that the DDMP method replaces the discontinuous derivative of the shape function $\nabla S_{i}$ with $\overline{\nabla S_{i}}$ defined in (13). $\overline{\nabla S_{i}}$ is continuous because the shape function $S_{j}(\boldsymbol{x})$ is continuous, and $\alpha(\boldsymbol{x})=0$ on cell boundaries, where the discontinuity in $\nabla S_{i}$ occurs.

Both the original MPM and the DDMP method use Riemann sums, but they are used for different purposes. In the original MPM, the Riemann sum is used essentially for numerical differentiation of the stress by summing over the products of the stresses and the gradients of the shape functions. The increase of the number of material points causes more frequent numerical noise generation as more material points can move across cell boundaries. Although the magnitude of the noise generated by each particle is reduced 
because the resulting discontinuity in the internal force is proportional to the particle volume, the overall effect on the numerical solution quality is very limited as shown in Fig. 3.

In the DDMP method, the Riemann sum is used to approximate the integral of the stress around a mesh node. The increase of the material points provides an improved accuracy in the calculation of the average nodal stress as required in (11), leading to a better solution quality. Although the first term in (13) is similar to the Riemann sum used in the MPM for numerical differentiation of the stress, its presence in DDMP is for the purpose of numerical stability, not for numerical differentiation. It comes from stress $\boldsymbol{A}$ defined in (8). The two terms in (8) almost cancel each other and $\boldsymbol{A}=O(\Delta x)^{2}$ independent of material points [13]. The presence of $\boldsymbol{A}$ eliminates a null space of stress in the force calculation and provides the effect of using staggered grids. For instance, in cases of a uniform mesh in a one-dimensional problems or a uniform rectangular mesh in a two-dimensional problem, if the stresses in the neighbor cells take values of equal magnitudes but opposite signs, the stress "checker boarding" situation, the second term in (7) vanishes. Without $\boldsymbol{A}$, the internal nodal force calculated using $\boldsymbol{\sigma}+\boldsymbol{\sigma}_{A}$ in (2) or from (12) is exactly zero, therefore provides no resistance to this spurious stress mode. With the presence of $\boldsymbol{A}$, while the second term of (8) also vanishes, the first term of (8), which results in the first term in (13), causes a nonzero internal force leading to a material motion reducing the magnitude of the spurious stress.

With this understanding of the DDMP method and the particle sum in (12), we find that the increase of material points provides a better approximation to the nodal stress as shown in (11) and a better solution quality as shown in Fig. 6.

In the next section we introduce a new method that has the accuracy equivalent to the use of many particles in the DDMP method but with the amount of computation greatly reduced. This new method incorporates the idea of particle domains of GIMP and CPDI, but without the need to track their shapes exactly. 


\section{DDMP with Sub-points}

In the Riemann sum (11), the length scale of the stress variation is determined by the physical problem, while the length scale of shape function is the cell size $\Delta x$, which is much smaller compared to the physical length scale in a reasonable calculation. The need for many material points in (11) arises not from the stress variation but from the variation of the shape functions over the cell size $\Delta x$. The new method originates from this observation and uses a small number of particles, say two particles per spatial dimension per cell, to adequately represent the stress variation over the physical length scale. We split each particle $(p)$ into a group of $\left(n_{p}\right)$ sub-particles or sub-points having the same stress as the original particle. These sub-points are distributed around the original material point. With these sub-points we re-write (12) as

$$
\boldsymbol{f}_{i}^{i n t}=-\sum_{p=1}^{N_{p}} \boldsymbol{\sigma}_{p} \cdot \sum_{s=1}^{n_{p}} v_{p s} \overline{\nabla S_{i}}\left(\boldsymbol{x}_{p s}\right)=-\sum_{p=1}^{N_{p}} \boldsymbol{\sigma}_{p} v_{p} \cdot \overline{\overline{\nabla S_{i}}}\left(\boldsymbol{x}_{p}\right)
$$

where $v_{p s}$ is the volume of sub-point $s$ belonging to original particle $p$ satisfying $\sum_{s=1}^{n_{p}} v_{p s}=$ $v_{p}, \boldsymbol{x}_{p s}$ is the position of the sub-point, and

$$
\overline{\overline{\nabla S_{i}}}\left(\boldsymbol{x}_{p}\right)=\frac{1}{v_{p}} \sum_{s=1}^{n_{p}} v_{p s} \overline{\nabla S_{i}}\left(\boldsymbol{x}_{p s}\right),
$$

is the sub-point volume weighted average of $\overline{\nabla S_{i}}$. The method of specifying volumes and positions of the sub-points will be discussed later. Equation (14) can be derived by following the derivations from (7) to (12). One can first treat all the sub-points as ordinary DDMP particles, calculate the internal force using (12), and then factor out stresses from the group of sub-points belonging to the same main particles $p$. With many sub-points, the approximation of (11) is better leading to enhanced quality of the numerical solution.

As number of sub-points approaches infinity, the new gradient of the shape function defined in (15) approaches $\int_{\Omega_{p}(t)} \overline{\nabla S_{i}} d v / v_{p}$, where $\Omega_{p}(t)$ is the particle domain. Although $\overline{\nabla S_{i}}$ is a function independent of the particles as defined in (13), direct evaluation of the integral is not an easy task, because the particle domain $\Omega_{p}(t)$ is a function of time. Approximately 
tracking the particle domain is a main task of the CPDI method. Unfortunately, the approximation introduced in the CPDI method does not satisfy the conservation properties required for high quality numerical solutions, but the scheme of approximately tracking the particle domains is useful in our new method introduced here. Before we discuss the use of the CPDI method further, let us first study conservation properties of this new method.

Relation (15) can be viewed as another modification to the gradient of the shape function. With this modified gradient of the shape function, we can still easily prove that

$$
\sum_{i}^{N} \boldsymbol{f}_{i}^{i n t}=\mathbf{0}
$$

because $\sum_{i}^{N} \overline{\nabla S_{i}}\left(\boldsymbol{x}_{p s}\right)=\mathbf{0}$ according to (30) in [13], where $N$ is number of mesh nodes. This relation shows that the internal forces sum to zero over the computational domain and ensures momentum conservation. Because the method of computing nodal mass is not changed in this new method, mass conservation is unaffected.

To consider energy conservation, we note that kinetic energy difference in a time step $n$ to $n+1$ can be calculated using (46) in [13]

$$
K^{n+1}-K^{n}=\Delta t \sum_{i=1}^{N} \boldsymbol{v}_{i}^{n+1 / 2} \cdot \sum_{\ell=1}^{N} C_{\ell i} \boldsymbol{f}_{\ell}^{i n t}
$$

after neglecting the boundary force and body force terms, where $C_{\ell i}$ is the force transfer coefficient $[13,14]$ and $\boldsymbol{v}_{i}^{n+1 / 2}=\left(\boldsymbol{v}_{i}^{L}+\boldsymbol{v}_{i}^{n}\right) / 2$ is the half time Lagrangian velocity, with $\boldsymbol{v}_{i}^{L}$ being the Lagrangian velocity obtained from (1).

Substituting (14) into (17), we find

$$
K^{n+1}-K^{n}=-\Delta t \sum_{p=1}^{N_{p}} v_{p} \boldsymbol{\sigma}_{p}: \overline{\nabla \boldsymbol{v}}^{n+1 / 2}\left(\boldsymbol{x}_{p}^{n}\right)
$$

where

$$
\overline{\nabla \boldsymbol{v}}^{n+1 / 2}\left(\boldsymbol{x}_{p}^{n}\right)=\sum_{\ell=1}^{N} \sum_{i=1}^{N} C_{\ell i} \boldsymbol{v}_{i}^{n+1 / 2} \overline{\overline{\nabla S_{\ell}}}\left(\boldsymbol{x}_{p}^{n}\right)=\frac{1}{v_{p}} \sum_{s=1}^{n_{p}} v_{p s}\left(\sum_{\ell=1}^{N} \overline{\nabla S_{\ell}}\left(\boldsymbol{x}_{p s}\right) \sum_{i=1}^{N} C_{\ell i} \boldsymbol{v}_{i}^{n+1 / 2}\right),
$$

and the second identity comes from the use of (15). In the first identity of (19), the velocity gradient at the main particle is calculated using the modified DDMP gradient 
of the shape function defined in (15), which is the average of the DDMP gradient of the shape function weighted by the sub-point volumes. This form is used in our numerical calculation of the velocity gradient. The second identity shows that this velocity gradient can also be regarded as the sub-point volume weighted average of the velocity gradients at the sub-points, since the quantity inside the brackets can be identified as the velocity gradient at the sub-point located at $\boldsymbol{x}_{p s}$.

The internal energy change due to the material deformation in the time step can be calculated as eq. (51) in [13]

$$
U^{n+1}-U^{n}=\sum_{p=1}^{N_{p}} v_{p} \boldsymbol{\sigma}_{p}: \dot{\boldsymbol{\varepsilon}}_{p} \Delta t+O\left[(\Delta t)^{2}\right],
$$

Comparing (18) and (20), if we use velocity gradient defined in (19) to calculate the strain rate. i.e. $\dot{\boldsymbol{\varepsilon}}_{p}=\left[\overline{\nabla \boldsymbol{v}}^{n+1 / 2}+\left(\overline{\nabla \boldsymbol{v}}^{n+1 / 2}\right)^{T}\right] / 2$, we can ensure energy conservation error is second order in both the time step size and the cell size.

The conservation properties discussed above are independent of specification of the positions and volumes of the sub-points. In other words, any method of specifying the volumes and positions of the sub-points will preserve the conservation properties of the DDMP method, which conserves mass and momentum exactly and conserves energy to the second order in both the time and spatial discretization [13]. Furthermore, because of this independence, there is no need to track the volume and position of the sub-points. The sub-point positions and volumes can be generated and assigned at every time step as long as $\sum_{s=1}^{n_{p}} v_{p s}=v_{p}$ is satisfied. In this way, the sub-points become simply integration points not Lagrangian material points. The original material points or particles are relieved from the role of integration points. The amount of computation related to a sub-point is negligible compared to that related to a material point. All we need with this new method is to generate the sub-points in the domain around the main material point. For instance, we can use the particle domain tracking method of CPDI and evenly distribute sub-points in parallelograms for two-dimensional or in parallelepipeds in three-dimensional problems.

In this method, the information about the domain of the main particles is used to place the sub-points, not to perform an exact calculation. The error on the determination of 
particle domain does not affect the conservation properties of the new method, although it affects solution accuracy. In cases of extreme deformation, where a local linear approximation to the displacement field fails, and severe gaps or overlaps by the parallelograms and parallelepipeds appear, to maintain accuracy one has the options to use CPDI2, the improved version of the CPDI method [15], which ensures no gap and overlap among the particle domains, or to directly track the position and volume evolution of the sub-points in the calculation. For cases of complex constitutive relations, if the second option is chosen, the computational cost of a sub-point is still very small compared to that of a main particle, because the stresses on the sub-points are not calculated directly using the constitutive relation, but copied from their main particles. These options are left for future exploration. In the rest of the present paper we limit ourselves to the study of the numerical properties of this new sub-point method when applied to one-dimensional shock waves. 

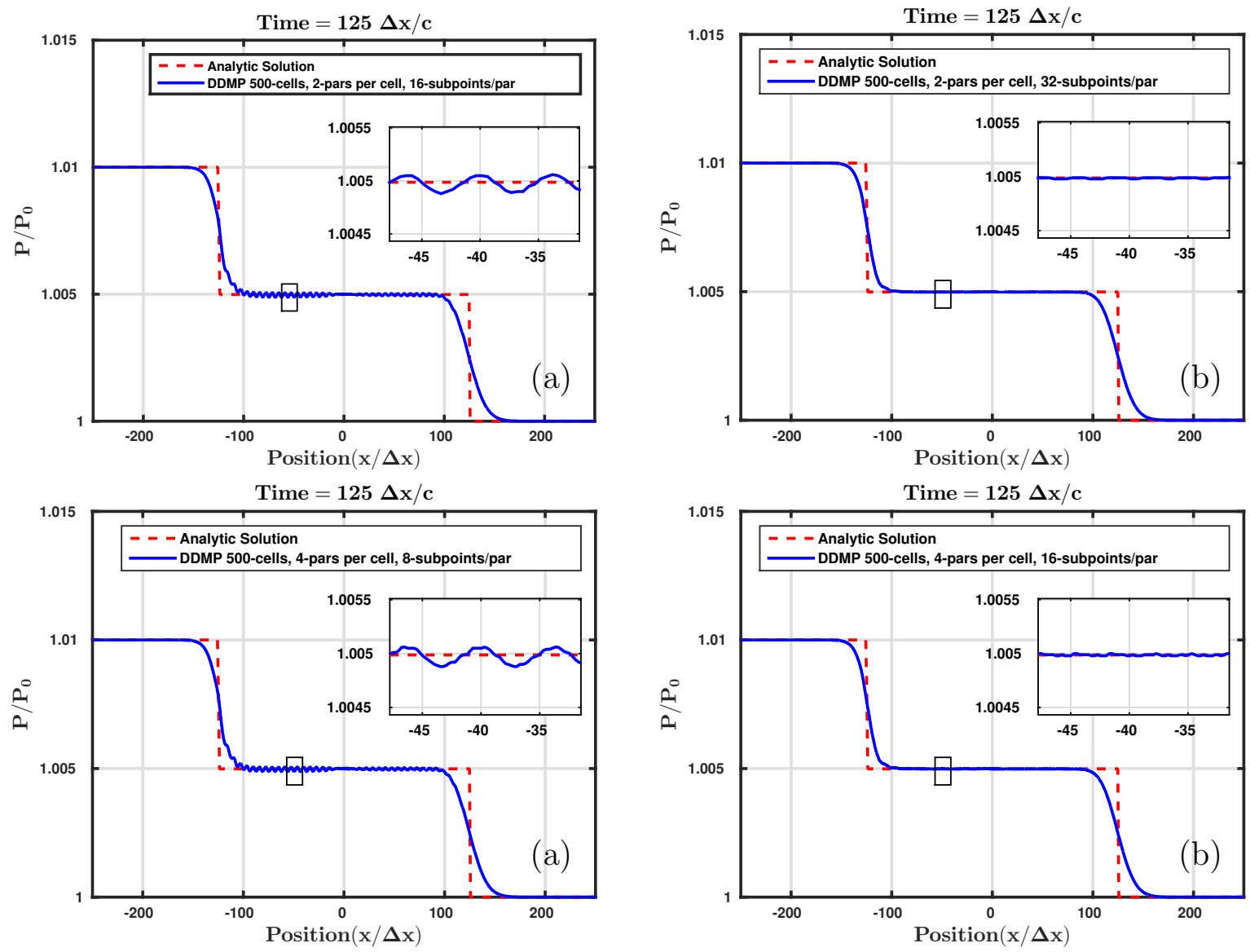

Figure 7: Results calculated using the sub-point method.

The results plotted in Fig. 7 are obtained by placing 2 or 4 particles per cell initially in the computational domain. Their volumes (actually, the lengths in this one-dimensional case) are updated using the velocity divergence at the particle location $\boldsymbol{x}_{p}$ calculated using the nodal velocity and the DDMP gradient of the shape function, $d v_{p} / d t=v_{p} \sum_{i=1}^{N} \boldsymbol{v}_{i}$. $\bar{\nabla} S_{i}\left(\boldsymbol{x}_{p}\right)$. Eight to 32 sub-points are evenly placed in each of the deformed particle domains at every time step. By comparison to Fig. 6, we find that the quality of the solution obtained from using this sub-point method is equivalent to that obtained using the number of main particles equal to the number of sub-points.

The results in Fig. 7 are obtained for a weak shock. In Fig. 8, we apply the new subpoint method to a much stronger shock. We compare the results calculated by initially placing 32 particles per cell using the DDMP method and the results obtained by using 

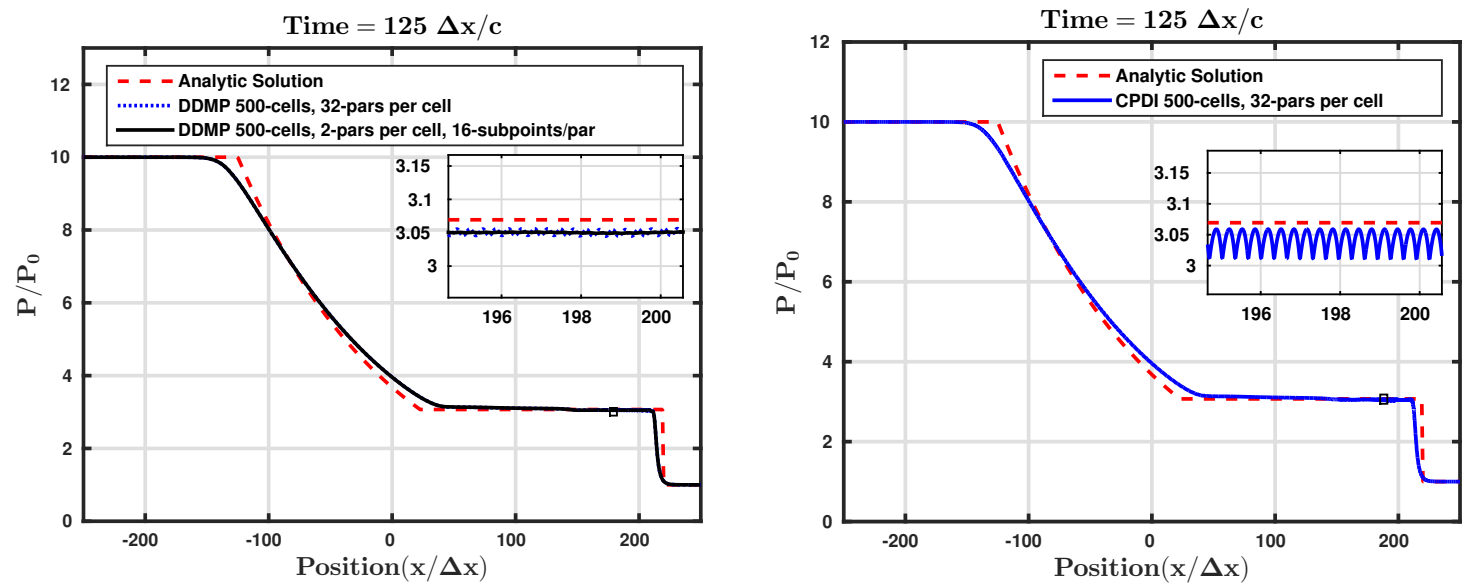

Figure 8: Comparison of strong shock results calculated using 2 particles per cell and 16 sub-points per particle with that obtained using 32 DDMP particles per cell (a). Strong shock results from CPDI method using 32 particles per cell (b).

only 2 DDMP particles initially in a cell with 16 sub-points per DDMP particle. Only very small differences are observed. For the calculation with 2 DDMP particles per cell, we plot the particle distribution in the region with expanded material in Fig. 9 at time $100 \Delta x / c$, earlier than the time $125 \Delta x / c$ for Fig. 8 . In this region there is less than one particle per cell. In this case, the tension instability has not happened because of the larger support of the modified gradient of the shape functions compared to that of the shape function, a feature inherent in DDMP. To test the limit, we also used one particle per cell in the initial particle placement. The calculation fails around time $25 \Delta x / c$ due to the tension instability.

In Fig. 8, the results of the CPDI method are also plotted on the right as a comparison. The sub-figure shows that the pressure noise exists in the CPDI result, although small relative to the large pressure difference in this strong shock problem. 


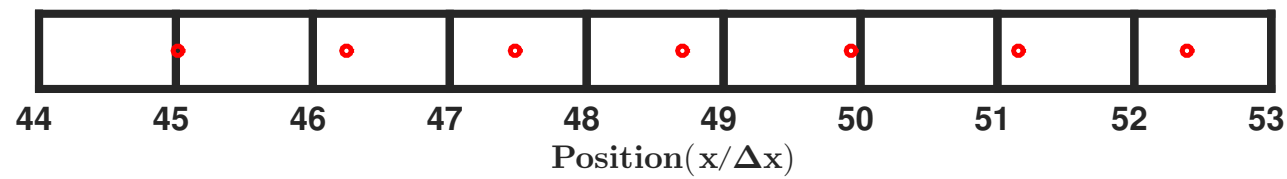

Figure 9: Distribution of particles in the region of expanded material in the strong shock calculation using the DDMP method with sub-points at time $100 \Delta x / c$. Initially two DDMP particles per cell are used. 16 sub-points per DDMP particle are used in this calculation.

\section{Conclusions}

The present paper shows that the original material point method cannot be used for weak isothermal shocks, because of the low accuracy of the Riemann sum used in the internal force calculation. Any attempt to increase the Riemann sum accuracy by increasing the number of particles is met with the cell crossing noise, because the use of more particles leads to more frequent cell crossing of the particles. Better numerical accuracy in the internal force calculation can be obtained by using GIMP and CPDI methods. However, these two methods fail to converge and generate noise as the number of particles increases. This non-convergence is caused by the use of a finite particle domain. As the number of particles increases, the particle domain is decreased, and the methods reduce to the original material point method, which suffers from cell-crossing noise. Although significant noise only occurs when a large number of particles $(>16)$ per cell are used, for materials undergoing a significant compression, the number of particles per cell can go well beyond 16 , leading to a compression instability.

The dual domain material point (DDMP) method is also used to study this simple example. Although the method converges as the number of particles increases, an impractically large amount of particles are needed to produce smooth results. The cause of the lack of smoothness is identified to be the insufficient accuracy in the calculation of the internal force integral by using the Riemann sum with a small amount of particles. A sub-point scheme is then introduced to increase the numerical integration accuracy. In this new scheme, the role of integration points is separated from the particles and given to the sub-points. In this way the accuracy of the numerical integration increases, and accurate 
results can be achieved with very small number of particles using the DDMP method. In this improved DDMP method, the sub-points can be generated and placed around the original DDMP particles at every time step. There is no need to track their history, therefore the computational cost incurred by these sub-points is negligible compared to that needed for the original DDMP particles. To place these sub-points, this new scheme needs an approximate particle domain. In one-dimensional cases, accurately tracking a particle domain is an easy task, but is very difficult for a multidimensional problem. In this subpoint scheme, the particle domain is not directly used in the integration for the internal force calculation; therefore errors, such as gaps and overlaps, of particle domains can be better tolerated than in the GIMP and CPDI methods. Furthermore, we have proved that placements of the sub-points do not alter conservation properties of the DDMP method, which conserves mass and momentum exactly and conserves energy to the second order of spatial and temporal discretization.

Although the numerical examples provided in this paper are one-dimensional, all derivations are in a multidimensional form. Extension of this sub-point method to multidimensional problems is rather straightforward. One can start from a DDMP code, and add the parallelograms (in two-dimension) and parallelepipeds (in three-dimension) tracking method of CPDI. Since both the DDMP and CPDI methods have been implemented for multidimensional problems, the only additional work to implement this sub-point method is to place sub-points evenly in the parallelograms or parallelepipeds and to calculate Riemann sums over these sub-points.

\section{Acknowledgments}

The authors would like to acknowledge many useful and in-depth discussions with Dr. Rick M. Rauenzahn of Los Alamos. This work was performed under the auspices of the United States Department of Energy. The Stockpile Safety and Surety Program, the Joint DoD/DOE Munitions Technology Development Program, and the ASC Program provided the financial support for this work. 


\section{References}

[1] F. H. Harlow, The particle-in-cell computing method for fluid dynamics, Methods Comput. Phys 3 (1964) 319.

[2] D. Sulsky, Z. Chen, H. L. Schreyer, A particle method for history-dependent materials, Computational Methods in Applied Mechanics and Engineering 118 (1994) 179-196.

[3] Z. Chen, Y. Han, S. Jiang, Y. Gan, T. D. Sewell, A multiscale material point method for impact simulation, Theoretical and Applied Mechanics Letters 2 (2012) 051003: $1-4$.

[4] Y. Lian, X. Zhang, F. Zhang, X. Cui, Tied interface grid material point method for problems with localized extreme deformation, International Journal of Impact Engineering 70 (2014) 50-61.

[5] R. Tian, Simulation at extreme-scale: Co-design thinking and practices, Arch Computat Methods Eng 21 (2014) 39-58.

[6] D. Sulsky, H. Schreyer, K. Peterson, R. Kwok, M. Coon, Using the material-point method to model sea ice dynamics, J. Geophysical Res. 112 (2007) C02S90.

[7] F. Hamad, D. Stolle, P. Vermeer, Modelling of membranes in the material point method with applications, Int. J. Numer. Anal. Meth. Geomech. 39 (2015) 833-853.

[8] P. C. Wallstedt, J. E. Guilkey, A weighted least squares particle-in-cell method for solid mechanics, Int. J. Numer. Meth. Engng 85 (2011) 1687-1704.

[9] S. G. Bardenhagen, E. Kober, The generalized interpolation material point method, Computer modeling in engineering and science 5(6) (2004) 477-495.

[10] A. Sadeghirad, R. Brannon, J. Burghardt, A convected particle domain interpolation technique to extend applicability of the material point method for problems involving massive deformations, Int. J. Numer. Meth. Engng. 286 (2011) 1435-1456. 
[11] D. Z. Zhang, Q. Zou, W. B. VanderHeyden, X. Ma, Material point method applied to multiphase flows, J. Comput. Phys 227 (2007) 3159-3173.

[12] D. Burgess, D. Sulsky, J. Brackbill, Mass matrix formulation of the flip particle-in-cell method, J. Comput. Phys 103 (1992) 1-15.

[13] D. Z. Zhang, X. Ma, P. T. Giguere, Material point method enhanced by modified gradient of shape function, J. Comput. Phys 230 (2011) 6379-6398.

[14] X. Ma, P. T. Giguere, B. Jayaraman, D. Z. Zhang, Distribution coefficient algorithm for small mass nodes in material point method, J. Comput. Phys. 229 (2010) 7819 7833.

[15] A. Sadeghirad, R. M. Brannon, J. E. Guilkey, Second-order convected particle domain interpolation (CPDI2) with enrichment for weak discontinuities at material interfaces, Int. J. Numer. Meth. Engng 95 (2013) 928-952. 